\title{
MIR346 Pre-miRNA
}

National Cancer Institute

\section{Source}

National Cancer Institute. MIR346 Pre-miRNA. NCI Thesaurus. Code C82823.

MIR346 is an oligoribonucleotide that is encoded by the human MIR346 gene and has a role in the regulation of gene expression. 